\title{
Solubilization of Phenol Derivatives in Polymer Micelles Formed by Cationic Block Copolymer
}

\author{
Irma Fuentes, ${ }^{1}$ Patricio Silva, ${ }^{2}$ Francisco Martinez, ${ }^{3}$ and Andrés F. Olea ${ }^{4}$ \\ ${ }^{1}$ Departamento de Ciencias Químicas y Recursos Naturales, Facultad de Ingeniería y Ciencias, Universidad de La Frontera, \\ 4811230 Temuco, Chile \\ ${ }^{2}$ Servicio de Laboratorios Clínicos, Facultad de Medicina, Pontificia Universidad Católica de Chile, Vicuña Mackenna 4686, \\ 7820436 Santiago, Chile \\ ${ }^{3}$ Departamento de Ciencia de los Materiales, Facultad de Ciencias Físicas y Matemáticas, Universidad de Chile, Avenida Tupper 2069, \\ 8370451 Santiago, Chile \\ ${ }^{4}$ Instituto de Ciencias Químicas Aplicadas, Facultad de Ingeniería, Universidad Autónoma de Chile, El Llano Subercaseaux 2801, \\ 8320325 Santiago, Chile
}

Correspondence should be addressed to Irma Fuentes; ifuentesl@uc.cl and Andrés F. Olea; andres.olea@uautonoma.cl

Received 31 January 2017; Revised 22 March 2017; Accepted 28 March 2017; Published 30 May 2017

Academic Editor: Piersandro Pallavicini

Copyright (c) 2017 Irma Fuentes et al. This is an open access article distributed under the Creative Commons Attribution License, which permits unrestricted use, distribution, and reproduction in any medium, provided the original work is properly cited.

\begin{abstract}
The aggregation of cationic block copolymers formed by polystyrene (PS) and poly(ethyl-4-vinylpyridine) (PS-b-PE4VP) was studied in aqueous solution. Diblock copolymers of PS and poly(4-vinylpyridine) were synthesized by sequential anionic polymerization using $\mathrm{BuLi}$ as initiator. Subsequently, the 4-vinylpyridine units were quaternized with ethyl bromide to obtain cationic PS-b-PE4VP block copolymers with different quaternization degree. The self-aggregation of cationic block copolymers was studied by fluorescence probing, whereas the morphology and size of polymer micelles were determined by transmission electronic microscopy. Results indicate that spherical micelles with sizes lower than $100 \mathrm{~nm}$ were formed, whereas their micropolarity decreases with increasing quaternization degree. The partition of phenols between the micellar and aqueous phase was studied by using the pseudo-phase model, and the results show that the partition coefficients increase with increasing length of the side alkyl chain and are larger for star micelles. These results are discussed in terms of three-region model.
\end{abstract}

\section{Introduction}

The self-assembly of amphiphilic block copolymers in selective solvents can result in the formation of a wide variety of aggregates, such as spheres, rods, and bilayers [1]. The specific morphology of the aggregate can be attributed to a balance of three contributions to the free energy of the system, namely, chain stretching in the core, interfacial energy, and repulsion between corona chains [2]. There are many factors that affect the above three terms, that is, block length of the copolymer [3], concentration of the precipitant, initial concentration of polymer [4], presence of additives [5], and common solvent used [6].

The type of micelle, formed in aqueous solution, depends on the relative proportion of the soluble and insoluble block in the polymer. In the case that the hydrophilic block is larger than the hydrophobic block, that is, the core is short compared with the diameter of the corona, a "star" micelle is formed. On the other hand, if the hydrophobic block length is larger than the length of the hydrophilic block, a "crew-cut" micelle is formed.

Aggregates of block copolymers in solution have potential industrial applications in fields, such as drug delivery $[7,8]$, cosmetic industry, catalysis, and separations [9].

In this work the partition of alkylphenols between the aqueous phase and the hydrophobic environment provided by the polymer aggregates was studied as a function of block size and charge density on the hydrophilic block. For this purpose two block copolymers formed by polystyrene and poly(4-vinylpyridine) PS-b-P4VP were synthesized with different lengths of both blocks. A subsequent reaction of the pyridine group with ethyl bromide has been used to generate 
positively charged polymer block (PE4VP) with different quaternization degrees. In this way, a variety of charged diblock copolymers PS-b-P4EVP with different block size and charge density were obtained.

\section{Materials and Methods}

Styrene and 4-vinyl pyridine (Aldrich) were dried by stirring over $\mathrm{CaH}_{2}$ overnight and distillated under reduced pressure. Ethyl bromide (Sigma-Aldrich) and pyrene (Molecular Probes) were used as received. Methanol, tetrahydrofuran, $\mathrm{N}, \mathrm{N}$-dimethylformamide (DMF), and chloroform HPLC grade (J. T. Baker) were used as received. Deionized water from a Millipore Milli-Q system (resistivity $18.2 \mathrm{M} \Omega \cdot \mathrm{cm}$ ) was used to prepare aqueous solutions.

\subsection{Synthesis of PS-b-PVP and PS-b-PE4VP Copolymers.} Diblock copolymers PS-b-P4VP were synthesized by sequential anionic polymerization using $\mathrm{n}-\mathrm{BuLi}$ as initiator [10]. The polymerization was carried out under inert atmosphere in a previous flamed glass reactor. In a typical reaction the initiator BuLi, dissolved in THF, is introduced into the reactor by using a syringe. After the solution has been cooled to $-78^{\circ} \mathrm{C}$, freshly distilled styrene was added, and the solution became yellow, which is the typical color of living polystyryl anions. The polymerization was allowed to proceed for $10 \mathrm{~min}$. After that, an aliquot of the reaction medium was withdrawn for analysis by size exclusion chromatography (SEC), in order to determine the degree of polymerization of the PS block. Subsequently, 4-vinylpyridine is added and immediately the solution color turns from yellow to orange. The polymerization is allowed to proceed for another $15 \mathrm{~min}$ at $-78^{\circ} \mathrm{C}$ and then stopped by adding methanol to quench the reaction and to precipitate the block copolymer.

The 4-vinylpyridine units of the PS-b-P4VP copolymer were reacted with ethyl bromide to obtain cationic PSb-PE4VP block copolymers with different percentages of quaternization. This reaction was carried out by dissolving PS-b-P4VP copolymer $(0.1 \mathrm{~g})$ in chloroform $(30 \mathrm{~mL})$ and using a 2-10-fold excess of ethyl bromide relative to 4vinylpyridine in the P4VP block. The mixture is refluxed with continuous stirring for 3 days. The PS-b-PE4VP copolymers were precipitated in hexane and dried in a vacuum oven at $40^{\circ} \mathrm{C}$ for 2 days.

Characterization. Size exclusion chromatography (SEC) was carried out with a Shimadzu LC-20AD apparatus equipped with a MZ-Gel SDplus column, a refractive index detector, and chloroform as the mobile phase. Standard polystyrenes were used for calibration; the number- and weight-average molecular weights and the polydispersity index of the polystyrene block were calculated using GPC Class VIP software. The composition was determined by FTIR and ${ }^{1} \mathrm{H}$ NMR measurements, using a Shimadzu FTIR IR Prestige 21, and a Bruker Avance 400 spectrometer, respectively. The degree of quaternization of P4VP blocks was determined by FTIR spectroscopy using characteristic bands of absorption of quaternized $\left(1640 \mathrm{~cm}^{-1}\right)$ and nonquaternized $\left(1600 \mathrm{~cm}^{-1}\right)$ repeating units [11].

\subsection{Preparation of Aqueous Dispersion of Diblock Copolymer} Micelles. Aqueous dispersion of diblock copolymers were prepared by using two different methodologies, depending on the relative length of the hydrophilic and hydrophobic blocks. Dialysis method was used for copolymer in which the hydrophobic block size is larger than the size of the hydrophilic block. As these copolymers cannot be dissolved directly in water, a certain amount of copolymer (2\%) was dissolved in DMF, a common solvent for both blocks, and stirred for $24 \mathrm{~h}$ at room temperature. Then the polymer solution was added to a volume of Milli-Q water $(18.2 \mathrm{M} \Omega \mathrm{cm})$ with volumetric dispensator with injection rate $0.15 \mathrm{~mL} / \mathrm{min}$. The mixture polymer/DMF/water was dialyzed against distilled water to remove DMF. A dialysis tube with a cutoff of $6-8 \mathrm{kD}$ (SpectrumLabs cellulose regenerate) was used and the water was changed four times a day for two days.

On the other hand, copolymers with hydrophilic block larger than the hydrophobic block were added directly to Milli-Q water $(18.2 \mathrm{M} \Omega \mathrm{cm})$ and the mixture was stirred for $24 \mathrm{~h}$ until complete dissolution.

2.3. Characterization of Micelles Dispersion. The morphology and diameter of aggregates formed PS-b-PE4VP copolymers micelles were assessed by transmission electron microscopy (TEM). A diluted sample is deposited on EM grids that had been precoated with a thin film of poly(vinylformaldehyde) plastic and then coated with carbon and staining the polymer with uranyl acetate. The images were obtained using a Philips Tecnai 12 Bio Tween operating at $80 \mathrm{kV}$ and the micrographs were analyzed using Sigma Scan Pro 4.0 software.

The critical micelle concentration (CMC) was determined by a photophysical method using pyrene as fluorescence probe. The intensities of the peaks at $339 \mathrm{~nm}$ and $333 \mathrm{~nm}$ in the excitation spectra were measured at different polymer concentrations ranging from $1 \times 10^{-9}$ to $1 \times 10^{-4} \mathrm{M}$, in molar monomer unit basis. The ratio of the intensities $I_{339} / I_{333}$ is plotted against logarithm of polymer concentration and the breaking point is associated with the CMC [12].

The micropolarity of copolymer micelles was obtained using the ratio of intensities of peaks appearing at $373 \mathrm{~nm}$ and $384 \mathrm{~nm}, I_{1} / I_{3}$, in the fluorescence spectra.

Pyrene excitation spectrum was obtained by fixing the emission wavelength at $390 \mathrm{~nm}$ and the excitation wavelength was scanned between 300 and $350 \mathrm{~nm}$. Emission spectrum of pyrene was registered between 350 and $500 \mathrm{~nm}$ and irradiation at $337 \mathrm{~nm}$. Pyrene stock solution was prepared in acetonitrile, and the final pyrene concentration was $5 \times$ $10^{-7} \mathrm{M}$. The excitation and emission spectrum of pyrene were obtained by using a Fluoromax 4 spectrofluorometer (Horiba Yvon) at room temperature.

\subsection{Measurement of Distribution of Phenol and Derivatives} into Diblock Copolymers Micelles. Incorporation of phenol and derivatives, into the polymeric micelles dispersion, was carried out by determining the distribution coefficient using 
a pseudo-phase model. In the pseudo-phase model the solubilization process is represented by the equilibrium:

$$
S_{W}+P_{M} \rightleftarrows S_{M},
$$

for which the equilibrium constant can be expressed on a molar concentration basis, $K_{S}$, as

$$
K_{S}=\frac{[S]_{M}}{[S]_{W}[P]_{M}} .
$$

$[S]_{M}$ and $[S]_{W}$ denote the molar concentration of the p-alkyl phenols in micellar polymeric and aqueous pseudo-phase, respectively. $[P]_{M}$ corresponds to the polymer concentration forming the micellar aggregates and $f_{W}$ and $f_{M}$ are the activity coefficient of the substrate in aqueous and micellar phases. On a mole fraction basis, the distribution coefficient can be expressed by

$$
K_{X}=\frac{X_{M}}{X_{W}}
$$

$X_{M}$ and $X_{W}$ are the mole fraction of substrate in the micellar polymeric and aqueous phases, respectively. In a dilute solution both quantities are related by $K_{X}=55.5 K_{S}$.

The distribution coefficients $\left(K_{S}\right)$ were determined by ultrafiltration method using an AMICON 810 cell with a polyethersulfone membrane (MWCO 5 KDalton). Aqueous solutions of PS-b-PE4VP $\left(6 \times 10^{-6} \mathrm{M}\right)$ containing different concentrations of p-alkylphenols were filtered, and the absorbances of the filtrate were measured at $275 \mathrm{~nm}$. The molar concentrations of phenols were obtained from a calibration curve. The values of $K_{S}$ were obtained from the initial slope of a plot of $[S]_{M}$ against $[S]_{W}$, according to (2), where the activity coefficient can be assumed to be unity.

\section{Results and Discussion}

3.1. Synthesis of PS-b-P4VP and PS-b-PE4VP. Two diblock copolymers of PS and P4VP were synthesized by anionic sequential polymerization. The molecular weight and dispersity index of PS homopolymer and PS-b-P4VP copolymers were determined by SEC. Chromatograms of synthesized block copolymers are shown in Figure 1.

The results indicate that two blocks of PS with degrees of polymerization 406 and 450 were obtained, and subsequently P4VP blocks of 546 and 47 repeat units were attached to these PS blocks, respectively. The dispersity indexes were 1.001 to 1.230 , suggesting a narrow distribution of molecular weight that is typical for anionic living polymerization. The formation of $\mathrm{PS}_{406}-\mathrm{b}-\mathrm{P} 4 \mathrm{VP}_{546}$ and $\mathrm{PS}_{450}-\mathrm{b}-\mathrm{P} 4 \mathrm{VP}_{47}$ was confirmed by ${ }^{1} \mathrm{H}-\mathrm{NMR}$ and FTIR spectroscopies and comparing with reported data.

To increase the amphiphilic character of PS-b-P4VP copolymers, a formal charge has been introduced into the P4VP block by reaction of pyridine ring with ethyl bromide. The fraction of pyridine ring converted to ethyl-4vinylpyridinium bromide was determined by FTIR using characteristic absorption bands of pyridine ring at 1600 at $\mathrm{cm}^{-1}$ and quaternized pyridine at $1640 \mathrm{~cm}^{-1}$ (Figure 2) [11].
TABLE 1: Values of the ratio $I_{1} / I_{3}$ of pyrene incorporated into micelles formed by $\mathrm{PS}_{406}$-b-PE4VP $\mathrm{VP}_{542}$ with different degrees of quaternization.

\begin{tabular}{lc}
\hline$\%$ quaternization & Ratio $I_{1} / I_{3}$ \\
\hline 40 & 1.4 \\
60 & 1.2 \\
80 & 1.1 \\
98 & 1.05 \\
\hline
\end{tabular}

The degree of quaternization was calculated by the following relation:

$\%$ Quaternization

$$
=\frac{\text { Absorbance } 1640 \mathrm{~cm}^{-1}}{\text { Absorbance } 1640 \mathrm{~cm}^{-1}+\text { Absorbance } 1600 \mathrm{~cm}^{-1}} \text {. }
$$

Block copolymer $\mathrm{PS}_{406}-\mathrm{b}-\mathrm{P} 4 \mathrm{VP}_{542}$ was quaternized at different degrees, that is, from $40 \%$ to $98 \%$ (see Table 1 ), whereas the degree of quaternization for $\mathrm{PS}_{450}$-b-P $4 \mathrm{VP}_{47}$ was $98 \%$. Therefore, the resulting polymers are ionomers carrying different number of charges.

3.2. Formation and Characterization of Polymer Micelles in Aqueous Solution. Aqueous dispersions of $\mathrm{PS}_{450}-\mathrm{b}-\mathrm{PE}_{4} \mathrm{VP}_{47}$ and $\mathrm{PS}_{406}-\mathrm{b}-\mathrm{PE} 4 \mathrm{VP}_{542}$ were prepared by dialysis and direct dissolution methods, respectively.

The aggregation of $\mathrm{PS}_{406}-\mathrm{b}-\mathrm{PE} 4 \mathrm{VP}_{542}$ chains to form polymer micelles was monitored by fluorescence measurements using pyrene as probe. It has been shown that photophysical properties of pyrene are strongly affected by the polarity of the medium where it is located [13]. Basically, in presence of micelles pyrene is solubilized into the hydrophobic environment provided by the micelle core. Thus, both excitation and emission spectrum of the molecular probe changes upon transfer of pyrene from aqueous environment to the nonpolar environment of the polymer micelle. These changes have been used to determine the critical micelle concentration of amphiphilic polymers [14-16]. In this work, the changes in the excitation spectrum of pyrene (Figure 3(a)) were used to determine the CMC of $\mathrm{PS}_{406}-\mathrm{b}-\mathrm{PE} 4 \mathrm{VP} \mathrm{P}_{542}$. In this method the ratio of intensities of bands at $333 \mathrm{~nm}$ and $339 \mathrm{~nm}, I_{339} / I_{333}$, is plotted as function of polymer concentration (Figure 3(b)), and the CMC is identified as the breaking point $[12,17]$. The value of CMC obtained by this method is in the range of $10^{-6}$ to $10^{-7} \mathrm{M}$, which is quite similar to the values reported for $\mathrm{PS}_{100}-\mathrm{b}-\mathrm{PE} 4 \mathrm{VP} \mathrm{P}_{100}(3 \times$ $\left.10^{-7} \mathrm{M}\right)$ [18] and $\mathrm{PS}_{86}-\mathrm{b}-\mathrm{PAA}_{390} \mathrm{CMC} 1,5 \times 10^{-7} \mathrm{M}$ [19]. These values of CMC are almost independent of the quaternization degree, which indicate that the high tendency to association at low concentration of copolymer chains is mainly due to the presence of a hydrophobic block such as polystyrene.

On the other hand, the effect of quaternization on the micropolarity of block copolymers micelles formed by $\mathrm{PS}_{406}{ }^{-}$ b-PE4VP ${ }_{542}$ was evaluated by fluorescence probing. The most used parameter is the ratio $I_{1} / I_{3}$ that has been used to determine polarity of different kinds of hydrophobic structures, such as micelles, vesicles, and polymeric aggregates. 


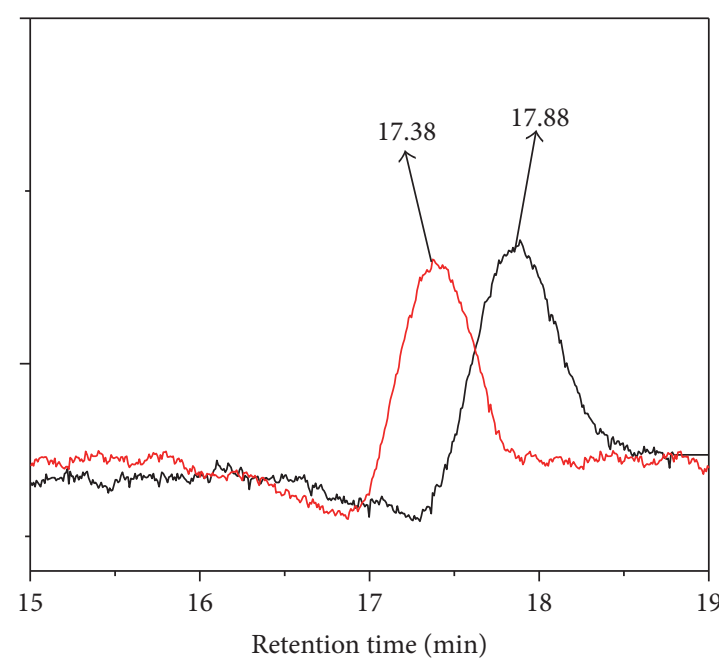

(a)

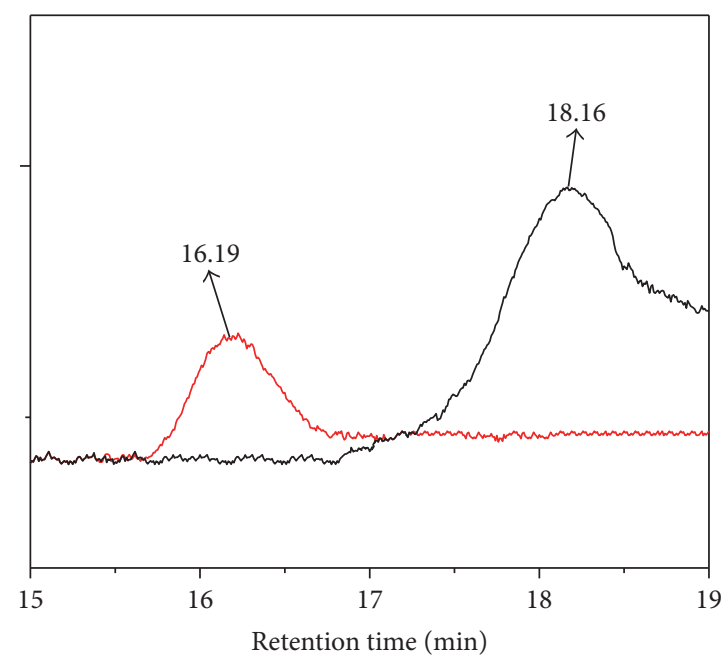

(b)

FIGURE 1: SEC chromatograms of PS (black lines) and PS-b-P4VP copolymers (red lines): (a) PS and PS ${ }_{450}-\mathrm{b}-\mathrm{P}_{4 V \mathrm{VP}_{47}}$; (b) PS and PS $406-\mathrm{b}-$ $\mathrm{P}_{4} \mathrm{VP}_{542}$.

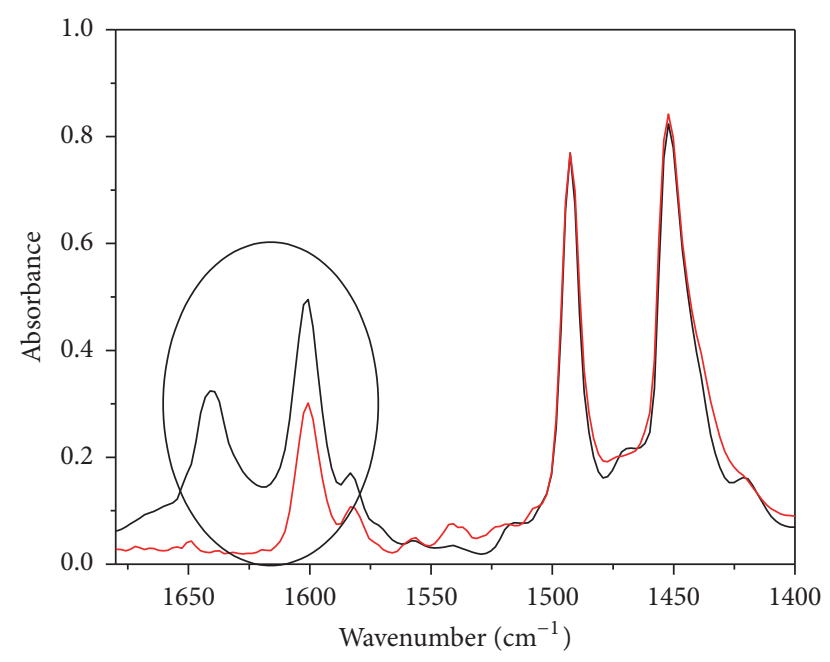

FIGURE 2: FTIR spectra of $\mathrm{PS}_{406}$-b-P4VP 542 (red line) and $\mathrm{PS}_{406}$-b$\mathrm{PE}_{4 \mathrm{VP}}{ }_{542}$ copolymer with $60 \%$ degree of quaternization (black line).

In aqueous solution the value of this ratio is 1.8 , whereas, in presence of PS-b-poly(ethylene oxide), PS-b-PEO, this ratio is 1.2 [18]. The values of the ratio $I_{1} / I_{3}$ in presence of cationic $\mathrm{PS}_{406}$-b-PE4VP $\mathrm{V}_{542}$ copolymer as a function of the degree of quaternization are shown in Table 1.

The data indicate that polarity of the micelle core decreases $\left(I_{1} / I_{3}\right.$ decreases) with increasing degree of quaternization. This behavior has been explained in terms of a change of the micellar structure induced by the variation of charge density into the hydrophilic block. Increasing the degree of quaternization of the pyridinium group generates an increment of the charge density in the corona of the aggregate and consequently a significant increase of the electrostatic repulsion between ethyl-4-vinylpyridinium bromide chains. To minimize this repulsion, the chains of PEVP tend to stretch forming a rigid corona and consequently a more compact hydrophobic core. This change of micellar structure might bring about a decrease in the amount of water associated with the micellar structure and therefore a decrease in the values of the ratio $I_{1} / I_{3}$.

To get an idea of the size and structure of micelles formed by PS-b-PE4VP images of aqueous solution of both copolymers were obtained by transmission electronic microscopy.

Images shown in Figure 4 indicate that polymer micelles are spherical with diameters lower than $100 \mathrm{~nm}$. It is also seen that there is a tendency of micelles to aggregate forming largest composite micelles.

3.3. Partition of Phenol and Derivatives between Aqueous and Micellar Phases. The partition of phenols between the polymer and aqueous phase was determined for $\mathrm{PS}_{450}$-b$\mathrm{P}_{4} \mathrm{VP}_{47}$ and $\mathrm{PS}_{406}-\mathrm{b}-\mathrm{PE} 4 \mathrm{VP}_{542}$ quaternized $98 \%$ and at concentrations well above the CMC. The distribution coefficients were obtained from the initial slope of the plots shown in Figure 5 according to (2), whereas $K_{X}$ were calculated with the relation $K_{X}=55.5 K_{S}$. Values of both distribution constants are summarized in Table 2.

The results show that the partition coefficients increase with increasing length of the side alkyl chain and are larger for micelles formed by $\mathrm{PS}_{406}-\mathrm{b}-\mathrm{PE} 4 \mathrm{VP}_{542}$. The effect of phenol structure on $K_{S}$ is in line with previous results obtained in normal micelles [20] and polymer micelles [21-23]. On the other hand, since both copolymers have PS blocks of almost identical length the micellar core sizes of their aggregates should also be similar. This conclusion is based on the assumption that the aggregation number of both micelles is the same, which is supported by the fact that the CMC values are exclusively determined by the size of the PS block, independently of the length and quaternization degree of the PE4VP block. The main difference between these polymer 


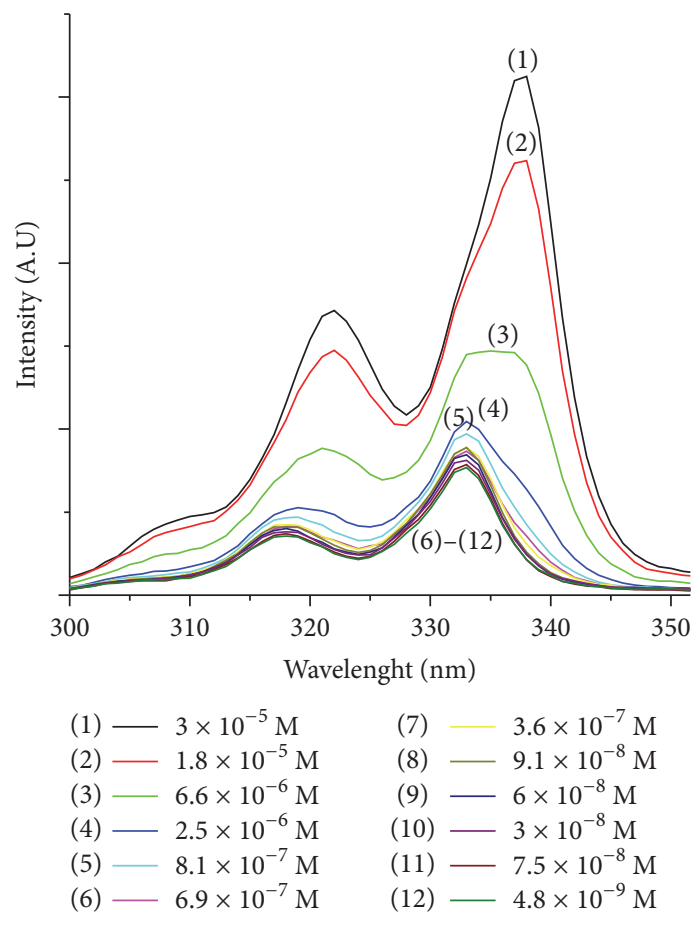

(a)

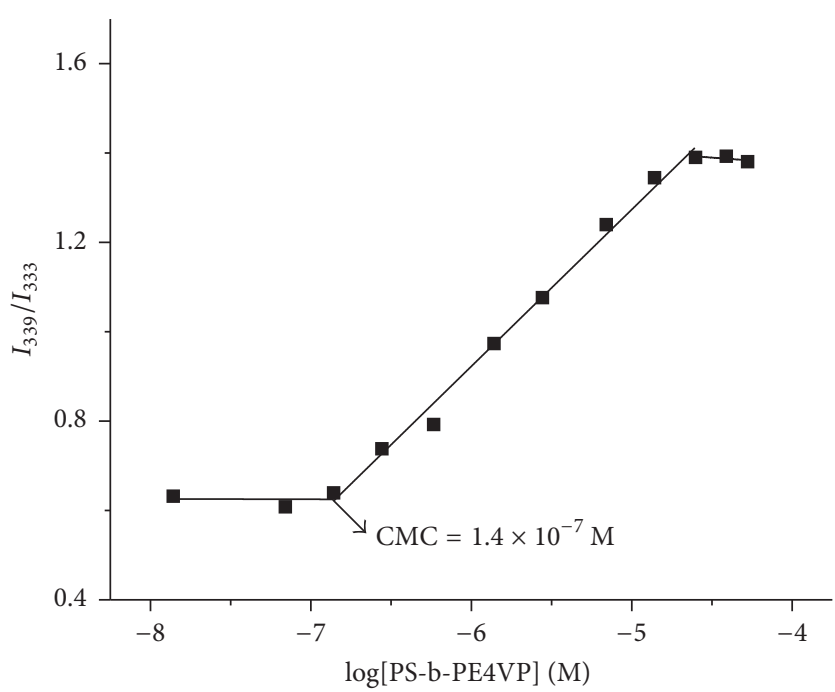

(b)

FIGURE 3: (a) Excitation spectrum of pyrene solubilized by $\mathrm{PS}_{450}-\mathrm{b}-\mathrm{PE} 4 \mathrm{VP} \mathrm{P}_{47}$ aqueous dispersion. (b) Determination of CMC of diblock copolymers using $I_{339} / I_{333}$ ratio from the fluorescence excitation spectrum of pyrene. Pyrene concentration $0.2 \mu \mathrm{M}$.

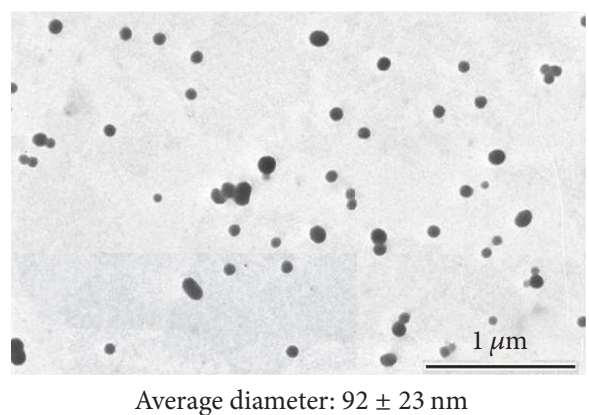

(a)

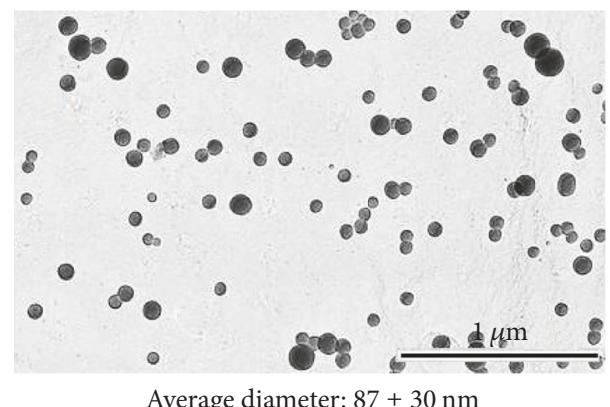

(b)

FIGURE 4: TEM images of diblock copolymers in aqueous dispersions: (a) $\mathrm{PS}_{450}$-b-P4VP 47 ; (b) $\mathrm{PS}_{406}-\mathrm{b}-\mathrm{PE}_{4 \mathrm{VP}} \mathrm{P}_{54}$.

micelles lays on the corona that is much larger for $\mathrm{PS}_{406}$-b$\mathrm{PE}_{4 \mathrm{VP}} \mathrm{F}_{42}$ than for $\mathrm{PS}_{450}-\mathrm{b}-\mathrm{PE} 4 \mathrm{VP}_{47}$. Thus, the higher values of $K_{S}$ for the former polymer can be explained in terms of solubilization of phenols in the inner corona. This threeregion model has been proposed to explain results of release kinetics and fluorescence quenching of aromatic molecules incorporated into aggregates formed by block copolymers of PS-b-poly(methacrylic acid) and PS-b-poly(acrylic acid). For these systems it was shown that an important fraction of solubilized molecules resides in the inner corona $[14,24]$.

In Table 2 are also given the values of the standard free energy of transfer from the aqueous phase to the pseudomicellar phase, which were calculated by the following relation:

$$
\Delta \mu_{t}^{0}=\mu_{M}^{0}-\mu_{W}^{0}=-R T \ln K_{X}
$$

It has been shown that $\Delta \mu_{t}^{0}$ can be separated in additive contributions from different constituent groups of the transferred substrate $[20,25]$ :

$$
\Delta \mu_{t}^{0}=\Delta \mu_{\mathrm{Ar}}^{0}+n_{\mathrm{C}} \Delta \mu_{\mathrm{C}}^{0}
$$

where $\Delta \mu_{\mathrm{Ar}}^{0}$ denotes the contribution of the parent aromatic group, $\Delta \mu_{C}^{0}$ is the incremental free energy per methylene group, and $n_{C}$ is the number of these groups attached to the parent group. Similar linear free energy relationships have been found in studies of partition of solutes between aqueous phase and organic solvents, micelles, and polymer micelles [20, 21, 25-27]. Plots of the values of $\Delta \mu_{t}^{0}$ against the number of carbon atoms in the alkyl group are almost linear for both polymer aggregates (see Figure 6). 


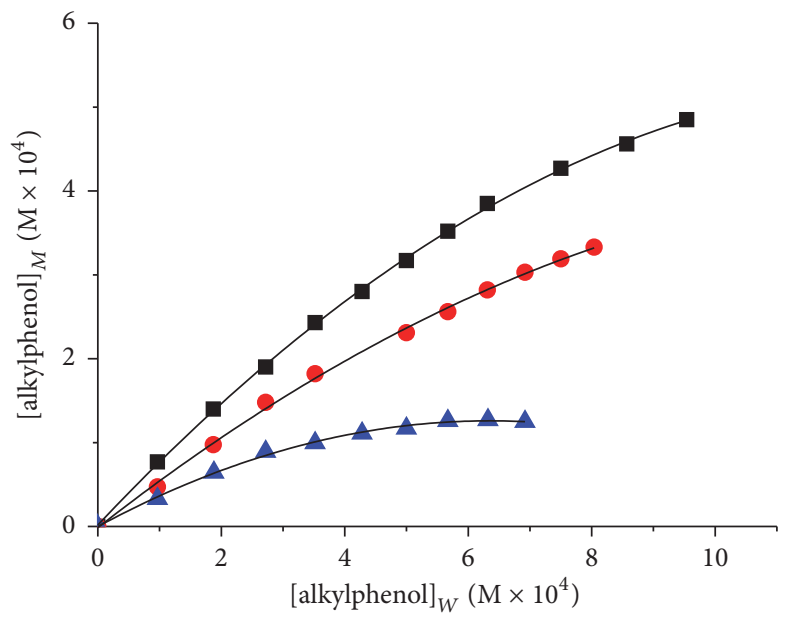

FIGURE 5: Distribution of phenol (blue triangle); p-cresol (red circle); and p-ethyl phenol (black square) between water and polymeric micelles of $\mathrm{PS}_{406}-\mathrm{b}-\mathrm{PE} 4 \mathrm{VP}_{542}$.

TABLE 2: Distribution constants and standard free energy obtained for transference of p-alkyl phenol between aqueous phase and polymer micelles formed by diblock copolymers.

\begin{tabular}{lcccccc}
\hline p-Alkylphenol & \multicolumn{3}{c}{$\mathrm{PS}_{450}-\mathrm{b}-\mathrm{PE} 4 \mathrm{VP} \mathrm{P}_{47}$} & \multicolumn{3}{c}{$\mathrm{PS}_{406}-\mathrm{b}-\mathrm{PE}_{\mathrm{VP}} \mathrm{V}_{52}$} \\
& $K_{S}$ & $K_{X}, \times 10^{4}$ & $\Delta \mu_{t}^{0}, \mathrm{~kJ} \mathrm{~mol}{ }^{-1}$ & $K_{S}$ & $K_{X}, \times 10^{4}$ & \multicolumn{2}{c}{$\Delta \mu_{t}^{0}, \mathrm{~kJ} \mathrm{~mol}^{-1}$} \\
\hline Phenol & 200 & 1.1 & -23.1 & 460 & -25.1 \\
p-Cresol & 250 & 1.4 & -23.6 & 780 & 4.3 & -26.4 \\
p-Ethylphenol & 270 & 1.5 & -23.8 & 985 & 5.5 & -27.0 \\
\hline
\end{tabular}

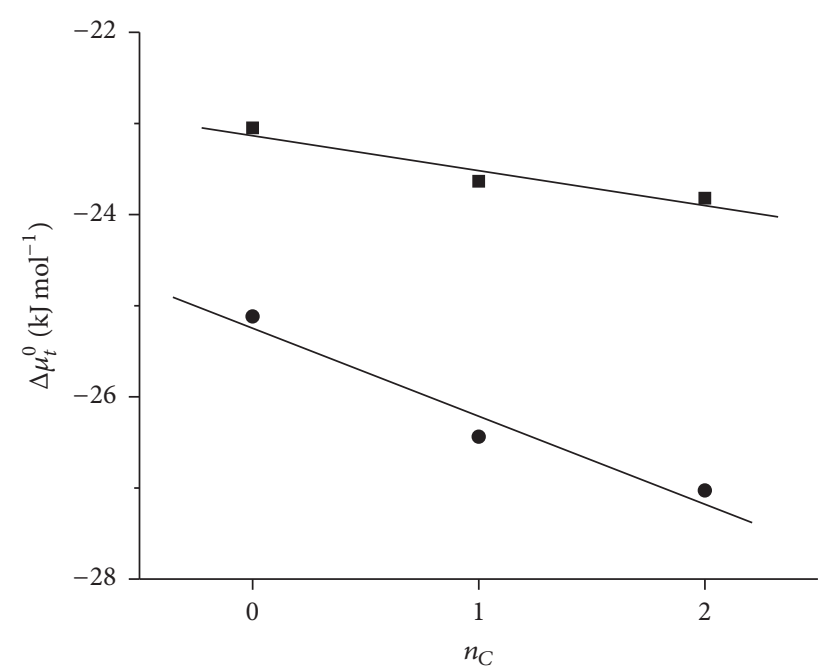

FIGURE 6: Standard free energy of transfer of p-alkyl phenols from water to polymer aggregates $\left(\Delta \mu_{t}^{0}\right)$, as a function of the number of carbon atoms on the alkyl chain. $\mathrm{PS}_{450}-\mathrm{b}-\mathrm{P} 4 \mathrm{VP}_{47}(\boldsymbol{\square})$ and $\mathrm{PS}_{406}$-b$\mathrm{PE}_{\mathrm{V}} \mathrm{VP}_{542}(\bullet)$.

The incremental free energy per methylene group, $\Delta \mu_{\mathrm{C}}^{0}$, obtained from the slopes are -0.39 and $-0.95 \mathrm{~kJ} \mathrm{~mol}^{-1}$ for $\mathrm{PS}_{450}-\mathrm{b}-\mathrm{PE}_{4 \mathrm{VP}} \mathrm{P}_{47}$ and $\mathrm{PS}_{406}$-b-PE4VP ${ }_{542}$, respectively. These values are much lower than that determined for transfer of methylene group from water to heptane $\left(-3.27 \mathrm{~kJ} \mathrm{~mol}^{-1}\right)$. These results suggest that alkyl chains are located into the micelle core of crew-cut micelles more deeply than in star micelles. On the other hand, the free energies of transfer of the aromatic moiety from water into polymer aggregates, $\Delta \mu_{\mathrm{Ar}}^{0}$, obtained from the intercept are -23.1 and $-25.2 \mathrm{~kJ} \mathrm{~mol}^{-1}$ for $\mathrm{PS}_{450}-\mathrm{b}-\mathrm{PE}_{4} \mathrm{VP}_{47}$ and $\mathrm{PS}_{406}$-b-PE4VP ${ }_{542}$, respectively. The values of $\Delta \mu_{\mathrm{Ar}}^{0}$ are larger than those of $\Delta \mu_{\mathrm{C}}^{0}$, which indicate that the location of p-alkylphenols in the polymer micelles formed by cationic diblock copolymers is mainly determined by the transfer of the aromatic moiety to the hydrophobic core, and in the case of $\mathrm{PS}_{406}-\mathrm{b}-\mathrm{PE}_{\mathrm{VPP}} \mathrm{P}_{52}$ the transfer to the inner corona. Interestingly, the values of $\Delta \mu_{\mathrm{Ar}}^{0}$ measured for these systems are similar to those determined for intramolecular micelles formed by sodium salts of poly(maleic acid-co-1-olefin) and much lower than those measured in other polymer and SDS micelles [21, 23]. This indicates that phenols incorporated to aggregates formed by cationic block copolymers are located in a more polar environment than that provided by normal micelles.

\section{Conclusions}

Block copolymers of PS and PVP were synthesized by sequential anionic polymerization. The resulting copolymers were quaternized by reaction with ethyl bromide leading to cationic block copolymers with different degrees of quaternization. The formation of polymer micelles was confirmed by fluorescence probing and transmission electronic microscopy. The polymer micelles have spherical 
shell-corona structures with diameters below $100 \mathrm{~nm}$. The distribution of phenol and alkyl derivatives was studied by using the pseudo-phase model and the partition constants and standard free energy of transfer were measured. The results indicate that transfer of the aromatic ring, from the aqueous phase to the micellar phase, is the main factor in the incorporation of these model compounds into the polymer aggregates. The higher values of $K_{S}$ and free energy of transfer found for star micelles formed by $\mathrm{PS}_{406}-\mathrm{b}-\mathrm{PE} 4 \mathrm{VP}_{542}$ are attributed to the phenol solubilization in the inner corona of these aggregates.

\section{Conflicts of Interest}

The authors declare that they have no conflicts of interest.

\section{Acknowledgments}

The authors gratefully acknowledge the financial support given by FONDECYT project 1130742.

\section{References}

[1] L. Zhang and A. Eisenberg, "Multiple morphologies and characteristics of 'crew-cut' micelle-like aggregates of polystyreneb-poly(acrylic acid) diblock copolymers in aqueous solutions," Journal of the American Chemical Society, vol. 118, no. 13, pp. 3168-3181, 1996.

[2] K. Letchford and H. Burt, "A review of the formation and classification of amphiphilic block copolymer nanoparticulate structures: micelles, nanospheres, nanocapsules and polymersomes," European Journal of Pharmaceutics and Biopharmaceutics, vol. 65, no. 3, pp. 259-269, 2007.

[3] A. Choucair, C. Lavigueur, and A. Eisenberg, "Polystyrene-bpoly(acrylic acid) vesicle size control using solution properties and hydrophilic block length," Langmuir, vol. 20, no. 10, pp. 3894-3900, 2004.

[4] H. Shen and A. Eisenberg, "Morphological phase diagram for a ternary system of block copolymer $\mathrm{PS}_{310}-\mathrm{b}-\mathrm{PAA}_{52} /$ dioxane $/ \mathrm{H}_{2} \mathrm{O}$," Journal of Physical Chemistry B, vol. 103, no. 44, pp. 9473-9487, 1999.

[5] L. Zhang and A. Eisenberg, "Morphogenic effect of added ions on crew-cut aggregates of polystyrene-b-poly(acrylic acid) block copolymers in solutions," Macromolecules, vol. 29, no. 27, pp. 8805-8815, 1996.

[6] L. Desbaumes and A. Eisenberg, "Single-solvent preparation of crew-cut aggregates of various morphologies from an amphiphilic diblock copolymer," Langmuir, vol. 15, no. 1, pp. 3638, 1999.

[7] A. V. Kabanov and V. Y. Alakhov, "Micelles of amphiphilic block copolymers as vehicles for drug delivery," in Amphiphilic Block Copolymers. Self-Assembly and Applications, P. Alexandridis and B. Lindman, Eds., pp. 347-376, Elsevier, Amsterdam, The Netherlands, 2000.

[8] M. Malstem, "Block copolymers in pharmaceutics," in Amphiphilic Block Copolymers. Self-Assembly and Applications, P. Alexandridis and B. Lindman, Eds., pp. 319-346, Elsevier, Amsterdam, The Netherlands, 2000.

[9] N. Hadjichristidis, S. Pispas, and G. Floudas, Block Copolymers, Synthetic Strategies, Physical Properties, and Applications, John Wiley \& Sons, Hoboken, NJ, USA, 2003.
[10] F. Bosse, H. P. Schreiber, and A. Eisenberg, "Specific adsorption of some styrene/vinylpyridine diblocks from selective solvents onto solid substrates-an NMR study," Macromolecules, vol. 26, no. 24, pp. 6447-6454, 1993.

[11] E. A. Lysenko, T. K. Bronich, E. V. Slonkina, A. Eisenberg, V. A. Kabanov, and A. V. Kabanov, "Block ionomer complexes with polystyrene core-forming block in selective solvents of various polarities. 2. Solution behavior and self-assembly in nonpolar solvents," Macromolecules, vol. 35, no. 16, pp. 6344-6350, 2002.

[12] M. Wilhelm, C.-L. Zhao, Y. Wang et al., "Poly(styrene-ethylene oxide) block copolymer micelle formation in water. A fluorescence probe study," Macromolecules, vol. 24, no. 5, pp. 10331040, 1991.

[13] K. Kalyanasundaram and J. K. Thomas, "Environmental effects on vibronic band intensities in pyrene monomer fluorescence and their application in studies of micellar systems," Journal of the American Chemical Society, vol. 99, no. 7, pp. 2039-2044, 1977.

[14] A. F. Olea, P. Silva, I. Fuentes, F. Martínez, and D. R. Worrall, "Probing solubilization sites in block copolymer micelles using fluorescence quenching," Journal of Photochemistry and Photobiology A: Chemistry, vol. 217, no. 1, pp. 49-54, 2011.

[15] I. Fuentes, B. Blanco-Fernandez, N. Alvarado et al., "Encapsulation of antioxidant gallate derivatives in biocompatible poly( $\epsilon$-caprolactone)-b-pluronic-b-poly( $\epsilon$-caprolactone $)$ micelles," Langmuir, vol. 32, no. 14, pp. 3331-3339, 2016.

[16] D. Y. Chu and J. K. Thomas, "Characterization of polymers by excited state techniques," in Photochemistry and Photophysics, J. F. Rabek, Ed., pp. 49-102, CRC Press, Boca Raton, Fla, USA, 1991.

[17] B. Urbano, P. Silva, A. F. Olea, I. Fuentes, and F. Martinez, "Selfassembly of triblock copolymers in aqueous solution," Journal of the Chilean Chemical Society, vol. 53, no. 2, pp. 1507-1510, 2008.

[18] E. A. Lysenko, T. K. Bronich, E. V. Slonkina, A. Eisenberg, V. A. Kabanov, and A. V. Kabanov, "Block ionomer complexes with polystyrene core-forming block in selective solvents of various polarities. 1. Solution behavior and self-assembly in aqueous media," Macromolecules, vol. 35, no. 16, pp. 6351-6361, 2002.

[19] M. Moffitt, K. Khougaz, and A. Eisenberg, "Micellization of ionic block copolymers," Accounts of Chemical Research, vol. 29, no. 2, pp. 95-102, 1996.

[20] L. Sepulveda, E. Lissi, and F. Quina, "Interactions of neutral molecules with ionic micelles," Advances in Colloid and Interface Science, vol. 25, pp. 1-57, 1986.

[21] A. F. Olea, R. G. Barraza, I. Fuentes, B. Acevedo, and F. Martinez, "Solubilization of phenols by intramolecular micelles formed by copolymers of maleic acid and olefins," Macromolecules, vol. 35, no. 3, pp. 1049-1053, 2002.

[22] A. F. Olea and C. Gamboa, "Solubilization of phenols in surfactant/polyelectrolyte systems," Journal of Colloid and Interface Science, vol. 268, no. 1, pp. 63-67, 2003.

[23] A. F. Olea, H. Carrasco, L. Espinoza, and B. Acevedo, "Solubilization of p-alkylphenols in Pluronics F-68 and F-127 micelles: partition coefficients and effect of solute on the aggregate structure," Journal of the Chilean Chemical Society, vol. 59, no. 2, pp. 2451-2454, 2014.

[24] Y. Teng, M. E. Morrison, P. Munk, S. E. Webber, and K. Procházka, "Release kinetics studies of aromatic molecules into water from block polymer micelles," Macromolecules, vol. 31, no. 11, pp. 3578-3587, 1998.

[25] C. Tanford, The Hydrophobic Effect, John Wiley \& Sons, New York, NY, USA, 2nd edition, 1988. 
[26] C. Gamboa, A. Olea, H. Rios, and M. Henriquez, "Association of alcohol with cationic micelles," Langmuir, vol. 8, no. 1, pp. 23-26, 1992.

[27] C. H. Salamanca, R. G. Barraza, B. Acevedo, and A. F. Olea, "Hydrophobically modified polyelectrolytes as potential drugs reservoirs of N-alkyl-nitroimidazoles," Journal of the Chilean Chemical Society, vol. 52, no. 1, pp. 1025-1029, 2007. 

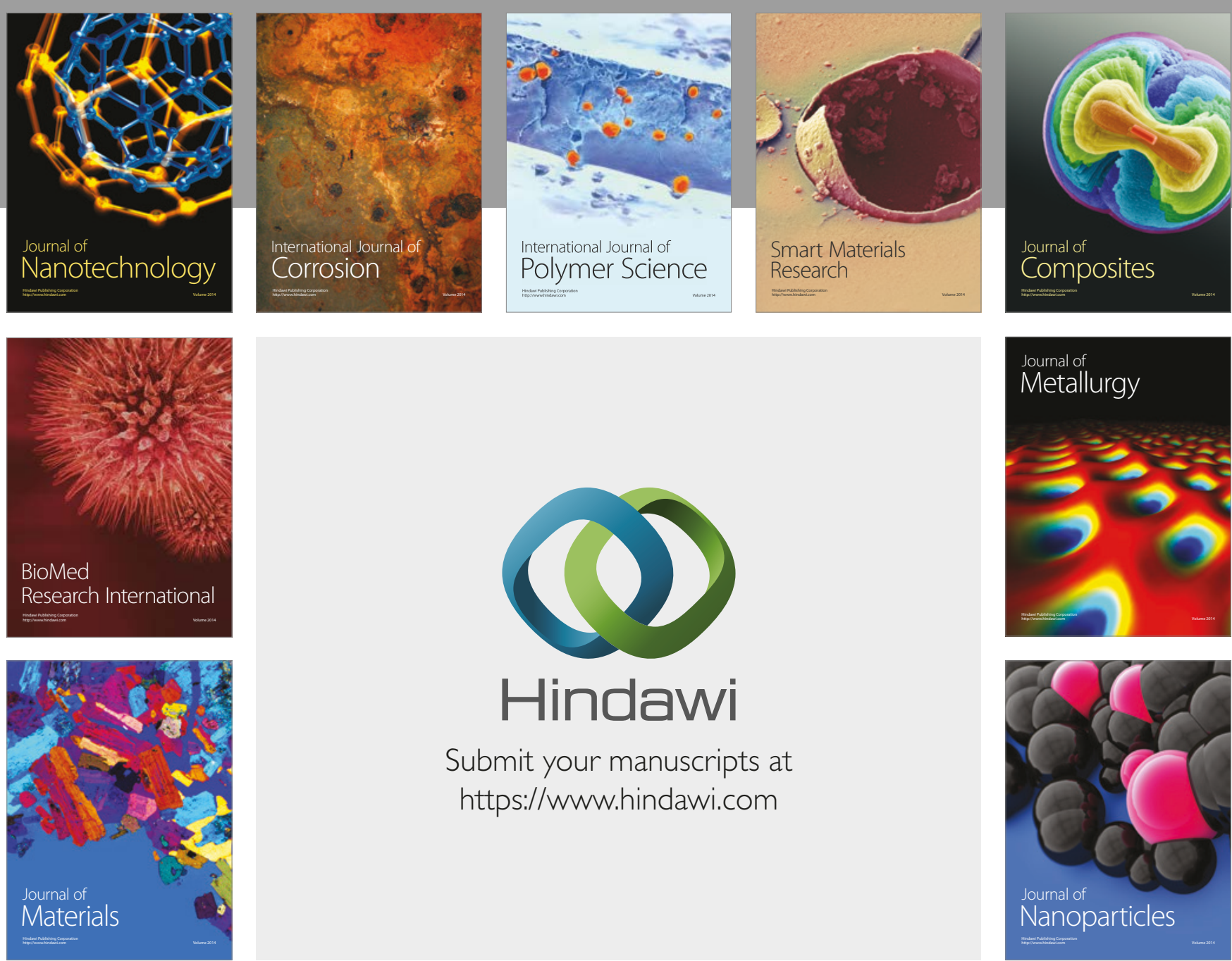

\section{Hindawi}

Submit your manuscripts at

https://www.hindawi.com
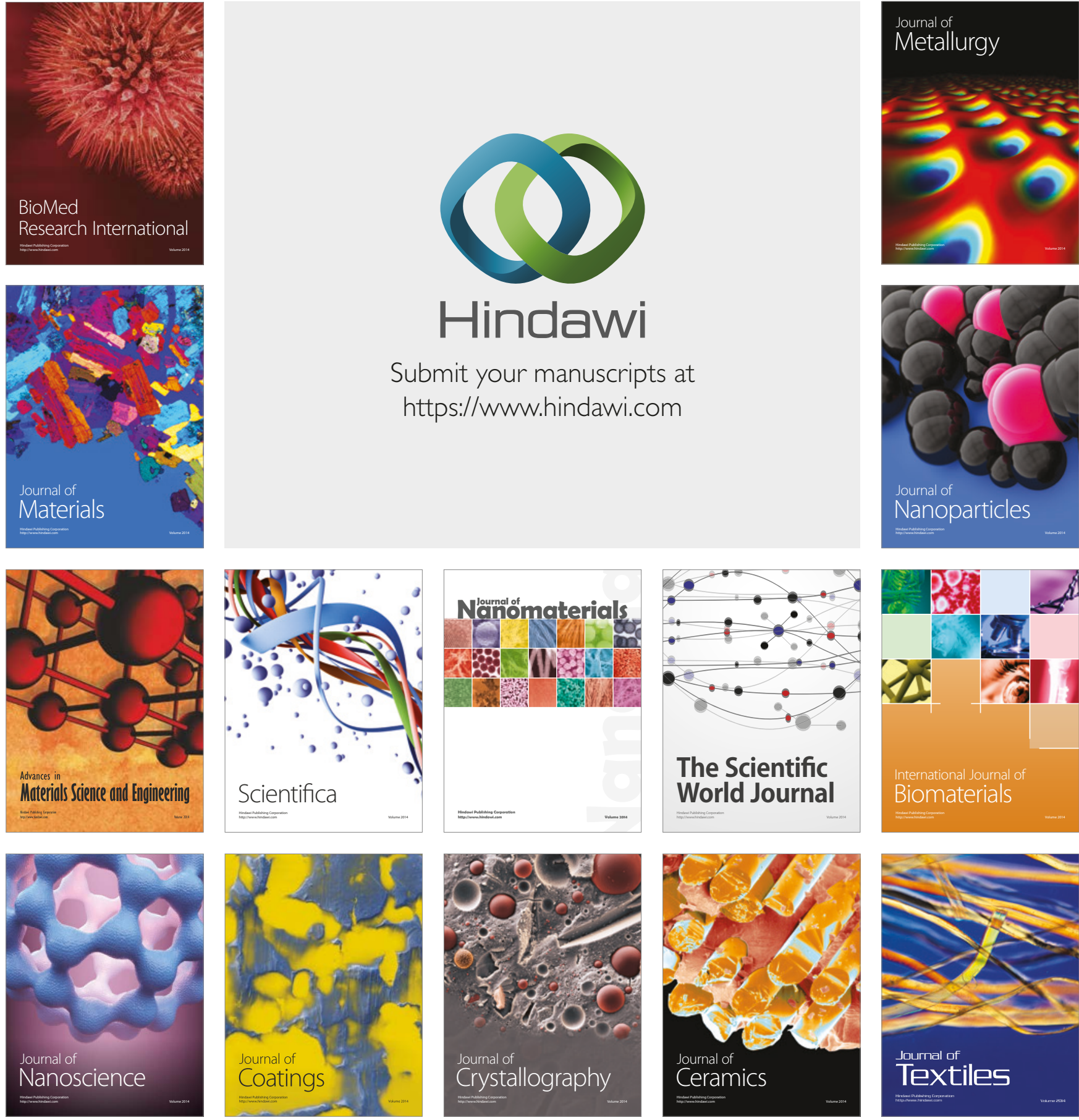

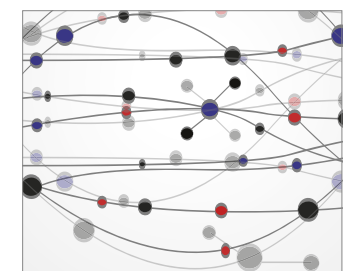

The Scientific World Journal
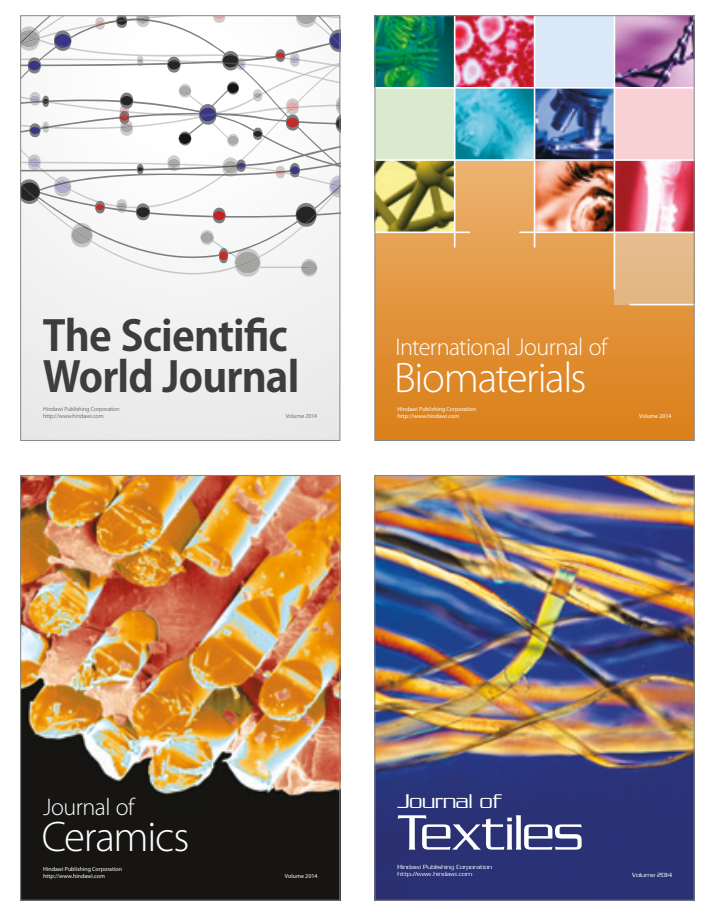